\title{
Seasonally Perturbed Prey-Predator Ecological System with the Beddington-DeAngelis Functional Response
}

\author{
Hengguo $\mathrm{Yu}^{1}$ and Min $\mathrm{Zhao}^{2}$ \\ ${ }^{1}$ School of Mathematics and Information Science, Wenzhou University, Wenzhou, Zhejiang 325035, China \\ ${ }^{2}$ School of Life and Environmental Science, Wenzhou University, Wenzhou, Zhejiang 325027, China
}

Correspondence should be addressed to Min Zhao, zmcn@tom.com

Received 1 October 2011; Accepted 2 November 2011

Academic Editor: Vladimir Gontar

Copyright (C) 2012 H. Yu and M. Zhao. This is an open access article distributed under the Creative Commons Attribution License, which permits unrestricted use, distribution, and reproduction in any medium, provided the original work is properly cited.

On the basis of the theories and methods of ecology and ordinary differential equation, a seasonally perturbed prey-predator system with the Beddington-DeAngelis functional response is studied analytically and numerically. Mathematical theoretical works have been pursuing the investigation of uniformly persistent, which depicts the threshold expression of some critical parameters. Numerical analysis indicates that the seasonality has a strong effect on the dynamical complexity and species biomass using bifurcation diagrams and Poincaré sections. The results show that the seasonality in three different parameters can give rise to rich and complex dynamical behaviors. In addition, the largest Lyapunov exponents are computed. This computation further confirms the existence of chaotic behavior and the accuracy of numerical simulation. All these results are expected to be of use in the study of the dynamic complexity of ecosystems.

\section{Introduction}

Population communities are embedded in periodically varying environments. Therefore, it is appropriate to identify the functional role that seasons play in the behavior of population communities [1]. This seasonal variation can cause changes in the dynamics of an ecological system. The study of ecological systems which are subjected to seasonal perturbations is important for both theoretical and experimental ecologists. This study should also take into account the intrinsic nature of environmental and seasonal perturbations [2]. The basic problem is to understand the relationship between the magnitude of the seasonal variations and the complexity of the system. Numbers of studies have been performed on the interactions between the seasons and the internal biological rhythms of simple prey-predator ecosystems [3-6]. These studies have shown that these interactions can 
have spectacular consequences, such as multiplicity of attractors, catastrophes, and chaos [7].

In recent decades, it has been demonstrated that complex dynamics can appear in continuous-time models with three or more species [8-12], and specifically that nonlinear dynamics, including cycles, quasicycles, and chaos, can occur in such biological systems. In this context, continuous-time and discrete-time systems [13-21] have been discussed extensively by a number of researchers. The Beddington-DeAngelis functional response is introduced by Beddington [22] and DeAngelis et al. [23], independently. The main difference of this functional response from other functional responses is that it contains an extra term presenting mutual interference by predators [24]. Although a direct link between the predators and preys cannot be established unless quantitative methods are used, the precious works clearly show that the amount of three species are often related, and a change in one species can cause a change in the others, especially predator. Thus, we apply BeddingtonDeAngelis functional response to describe their relationship with sufficient accuracy in this paper.

This paper considers a seasonally perturbed prey-predator system with the Beddington-DeAngelis functional response, which can be described by the following differential equations:

$$
\begin{aligned}
\frac{d x}{d t} & =r_{1}(t) x\left(\frac{k_{2}-x}{k_{1}-x}\right)-\frac{a_{1} \beta x y}{b_{1}+x+c_{1} y}-\frac{a_{2} x z}{b_{2}+x+c_{2} z} \\
\frac{d y}{d t} & =\left(\frac{e_{1} a_{1} \beta x}{b_{1}+x+c_{1} y}-m_{1}(t)\right) y, \\
\frac{d z}{d t} & =\left(\frac{e_{2} a_{2} x}{b_{2}+x+c_{2} z}-m_{2}(t)\right) z, \\
r_{1}(t) & =r_{1}\left(1+\varepsilon_{1} \sin (\omega t)\right) \\
m_{1}(t) & =m_{1}\left(1+\varepsilon_{2} \sin (\omega t+\varphi)\right) \\
m_{2}(t) & =m_{2}\left(1+\varepsilon_{3} \sin (\omega t+\varphi)\right)
\end{aligned}
$$

where $x(t), y(t)$, and $z(t)$ are the densities of one prey and two predators at time $t$, respectively, $a_{i}(i=1,2)$ are the cropping rates, $e_{i}(i=1,2)$ denote the efficiency with which preys are converted by new predators, $r_{1}(t) k_{2}$ is the carrying capacity of prey $x$, and $k_{1}$ is the value of the limiting prey. In other words, $k_{1}$ is the theoretical carrying capacity under ideal conditions if there is no wastage in preys, which is impossible in reality. $\left(k_{2} / k_{1}\right)(x(t) \leq$ $k_{2}<k_{1}$ ) expresses the efficiency of nutrient utilization by a species and has a value between zero and one. If the ratio approaches unity, the efficiency is high; a lower ratio indicates that population increase is quickly restricted by the limiting prey. $b_{i}(i=1,2)$ are saturation constants, and $c_{i}(i=1,2)$ are scaling factors expressing the impact of predator interference, and $\beta$ is the relative superiority of predator $y$. Without loss of generality, it can be assumed that $\beta>1$; accordingly, a predator with density $d z / d t$ is an inferior predator, while a predator with density $d y / d t$ is a superior predator because the ratio $(d y / d t) /(d z / d t) \propto \beta^{t}$ increases with time. $r_{1}, m_{1}$, and $m_{2}$ are the average values of $r_{1}(t), m_{1}(t)$, and $m_{2}(t)$, respectively, and $\omega$ is the angular frequency of the fluctuations caused by seasonality. The parameters $\varepsilon_{i}(i=1,2,3)\left(0 \leq \varepsilon_{i} \leq 1\right)$ represent the degree of seasonality, and $r_{1} \varepsilon_{1}, m_{1} \varepsilon_{2}$, and $m_{2} \varepsilon_{3}$ are 
the magnitudes of the perturbations in $r_{1}(t), m_{1}(t)$, and $m_{2}(t)$, respectively [25]. Finally, the parameter $\varphi$, where $0 \leq \varphi \leq 2 \pi$, can be interpreted as the difference in phase angle between the seasonality of the above three parameters. Clearly, when $\varphi=0$, there is synchronous variation in the intrinsic growth rate and death rate, while when $\varphi=\pi$, the variation is antisynchronous. In this paper, only three values of phase angles will be considered: $\varphi=0, \pi / 2$, and $\pi$.

\section{Mathematical Analysis}

Definition 2.1. System (1.1) is said to be uniformly persistent if, for any positive solution $(x(t), y(t), z(t))$ of system (1.1), there exist positive constants $m_{x}, m_{y}, m_{z}, M_{x}, M_{y}, M_{z}$, and $T>0$ such that $m_{x} \leq x(t) \leq M_{x}, m_{y} \leq y(t) \leq M_{y}$, and $m_{z} \leq z(t) \leq M_{z}$ for $t \geq T$.

Lemma 2.2 (see [26]). If $a>0, b>0,(d h(t) / d t) \leq(\geq) h(t)(b-a h(t))$, and $h(0)>0$, then, for any small constant $\varepsilon>0$, there exists a positive constant $T$ such that $h(t) \leq(b / a)+\varepsilon(h(t) \geq(b / a)-\varepsilon)$, for $t \geq T$.

Lemma 2.3 (see [27]). If $a>0, b>0,(d h(t) / d t) \leq(\geq) b-a h(t)$, and $h(0)>0$, then $h(t) \leq(\geq)$ $(b / a)+(h(0)-(b / a)) e^{-a t}$ for $t \geq 0$.

Now we can obtain the threshold expression of some critical parameters under the condition of all species persistence.

Theorem 2.4. There exist positive constants $M_{x}, M_{y}$, and $M_{z}$ for any positive solution $(x(t), y(t)$, and $z(t))$ of system (1.1) with all sufficiently large $t$.

Proof. Assume that $(x(t), y(t), z(t))$ is an arbitrary positive solution of system (1.1), then the first equation of system (1.1) can yield

$$
\begin{aligned}
\frac{d x(t)}{d t} & \leq r_{1}(t) x(t) \frac{k_{2}-x(t)}{k_{1}-x(t)} \leq r_{1}(t) x(t) \frac{k_{2}-x(t)}{k_{1}-k_{2}} \\
& \leq \frac{r_{1} k_{2}\left(1+\varepsilon_{1}\right)}{k_{1}-k_{2}} x(t)-\frac{r_{1}\left(1+\varepsilon_{1}\right)}{k_{1}-k_{2}} x^{2}(t) .
\end{aligned}
$$

From Lemma 2.2, we get that, for any small positive constant $\varepsilon$, there exists a constant $T_{1}>0$ such that $x(t) \leq k_{2}+\varepsilon:=M_{x}$ for $t \geq T_{1}$.

Defining that $V(t)=x(t)+\left(y(t) / e_{1}\right)+\left(z(t) / e_{2}\right)$, then

$$
\begin{aligned}
\dot{V}(t) & =\dot{x}(t)+\frac{\dot{y}(t)}{e_{1}}+\frac{\dot{z}(t)}{e_{2}} \\
& =r_{1}(t) x(t) \frac{k_{2}-x(t)}{k_{1}-x(t)}-\frac{m_{1}(t)}{e_{1}} y(t)-\frac{m_{2}(t)}{e_{2}} z(t) \\
& \leq \frac{r_{1} k_{2}\left(1+\varepsilon_{1}\right)}{k_{1}-k_{2}} x(t)-\frac{r_{1}\left(1-\varepsilon_{1}\right)}{k_{1}-k_{2}} x^{2}(t)-\frac{m_{1}\left(1-\varepsilon_{2}\right)}{e_{1}} y(t)-\frac{m_{2}\left(1-\varepsilon_{3}\right)}{e_{2}} z(t) .
\end{aligned}
$$


Let $0<L_{1}<\min \left\{m_{1}\left(1-\varepsilon_{2}\right), m_{2}\left(1-\varepsilon_{3}\right)\right\}$, then

$$
\begin{aligned}
\dot{V}(t)+L_{1} V(t) \leq & \left(\frac{r_{1} k_{2}\left(1+\varepsilon_{1}\right)}{k_{1}-k_{2}}+L_{1}\right) x(t)-\frac{r_{1}\left(1-\varepsilon_{1}\right)}{k_{1}-k_{2}} x^{2}(t) \\
& +\frac{L_{1}-m_{1}\left(1-\varepsilon_{2}\right)}{e_{1}} y(t)+\frac{L_{1}-m_{2}\left(1-\varepsilon_{3}\right)}{e_{2}} z(t) .
\end{aligned}
$$

Thus $\dot{V}(t)+L_{1} V(t)$ is bound. Set $L_{2}=\left[r_{1}\left(1+\varepsilon_{1}\right)+\left(k_{1}-k_{2}\right) L_{1}\right]^{2} /\left(4 r_{1}\left(1-\varepsilon_{1}\right)\left(k_{1}-k_{2}\right)\right)$ such that $\dot{V}(t) \leq-L_{1} V(t)+L_{2}$. According to Lemma 2.3, $V(t) \leq\left(L_{2} / L_{1}\right)+\left(V(0)-\left(L_{2} / L_{1}\right)\right) e^{-L_{1} t}$. Therefore $V(t)$ is ultimately bounded, and it follows that each positive solution of system (1.1) is uniformly ultimately bounded. Hence, there exist two positive $M_{y}, M_{z}\left(k_{2}+\left(M_{y} / e_{1}\right)+\right.$ $\left.\left(M_{z} / e_{2}\right) \leq\left[r_{1}\left(1+\varepsilon_{1}\right)+\left(k_{1}-k_{2}\right) L_{1}\right]^{2} /\left(4 r_{1} L_{1}\left(1-\varepsilon_{1}\right)\left(k_{1}-k_{2}\right)\right)\right)$ and $T_{2}$ such that $y(t) \leq M_{y}$ and $z(t) \leq M_{z}$, for $t \geq T_{2}$. This completes the proof.

Theorem 2.5. If $W_{1}>0, W_{2}>0$ and $W_{3}>0$, then system (1.1) is uniformly persistent.

Proof. From Theorem 2.4, it is obvious that there exist positive constants $M_{x}, M_{y}, M_{z}$, and $T_{3}=\max \left\{T_{1}, T_{2}\right\}>0$ such that $x(t) \leq M_{x}, y(t) \leq M_{y}, z(t) \leq M_{z}$ for $t \geq T_{3}$.

On the other hand, we get from system (1.1) that

$$
\begin{aligned}
\frac{d x(t)}{d t} & \geq r_{1}(t) x(t) \frac{k_{2}-x(t)}{k_{1}}-\frac{a_{1} \beta M_{y}}{b_{1}} x(t)-\frac{a_{2} M_{z}}{b_{2}} x(t) \\
& \geq\left(\frac{r_{1} k_{2}\left(1-\varepsilon_{1}\right)}{k_{1}}-\frac{a_{1} \beta M_{y}}{b_{1}}-\frac{a_{2} M_{z}}{b_{2}}\right) x(t)-\frac{r_{1}\left(1+\varepsilon_{1}\right)}{k_{1}} x^{2}(t)
\end{aligned}
$$

Then it follows from Lemma 2.2 that if $W_{1}=\left(r_{1} k_{2}\left(1-\varepsilon_{1}\right) / k_{1}\right)-\left(a_{1} \beta M_{y} / b_{1}\right)-\left(a_{2} M_{z} / b_{2}\right)>$ 0 , there is a positive constant $T_{4}$ such that $x(t) \geq\left(\left(r_{1} k_{2} b_{1} b_{2}\left(1-\varepsilon_{1}\right)-a_{1} \beta b_{2} k_{1} M_{y}-\right.\right.$ $\left.\left.a_{2} k_{1} b_{1} M_{z}\right) / r_{1} b_{1} b_{2}\left(1+\varepsilon_{1}\right)\right)-\varepsilon:=m_{x}$ for $t \geq T_{4}$.

From the second equation of system (1.1), we can obtain that $(d y(t) / d t) \geq$ $\left(\left(e_{1} a_{1} \beta m_{x} /\left(b_{1}+k_{2}+c_{1} M_{y}\right)\right)-m_{1}\left(1+\varepsilon_{2}\right)\right) y(t)$. It is obvious to know that if $W_{2}=\left(\left(e_{1} a_{1} \beta m_{x} /\left(b_{1}+\right.\right.\right.$ $\left.\left.\left.k_{2}+c_{1} M_{y}\right)\right)-m_{1}\left(1+\varepsilon_{2}\right)\right)>0$, there are two positive $T_{5}$ and $m_{y}$ such that $y(t) \geq m_{y}$ for $t \geq T_{5}$.

From the third equation of system (1.1), we can obtain that $(d z(t) / d t) \geq$ $\left(\left(e_{2} a_{2} m_{x} /\left(b_{2}+k_{2}+c_{2} M_{z}\right)\right)-m_{2}\left(1+\varepsilon_{3}\right)\right) z(t)$. It is obvious to know that if $W_{3}=\left(\left(e_{2} a_{2} m_{x} /\left(b_{2}+\right.\right.\right.$ $\left.\left.\left.k_{2}+c_{2} M_{y}\right)\right)-m_{2}\left(1+\varepsilon_{3}\right)\right)>0$, there are two positive constants $T_{6}$ and $m_{z}$ such that $z(t) \geq m_{z}$ for $t \geq T_{6}$. Let $T=\max \left\{T_{3}, T_{4}, T_{5}, T_{6}\right\}$ then we have $m_{x} \leq x(t) \leq M_{x}, m_{y} \leq y(t) \leq M_{y}$, and $m_{z} \leq z(t) \leq M_{z}$ for $t \geq T$. This completes the proof.

By using the theoretical analysis, we obtain the threshold expression of some critical parameters under the condition of all species persistence, which in turn provides a theoretical basis for the numerical simulation.

\section{Simulation Analysis and Results}

To study complex dynamics of the prey-predator system with seasonality, the solution of the system (1.1) with initial conditions is obtained numerically for the biologically feasible range of parametric values. In order to investigate the interplay among seasonal perturbation 


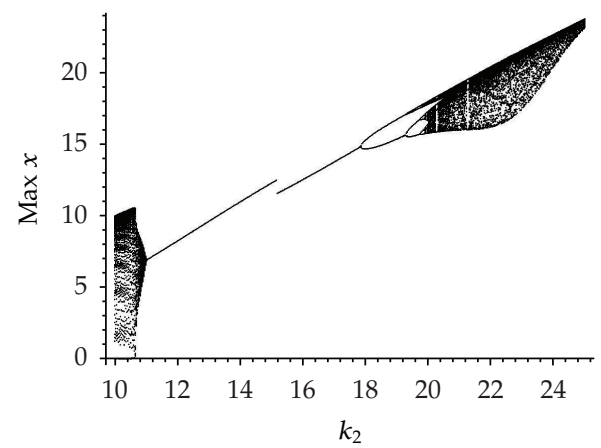

(a)

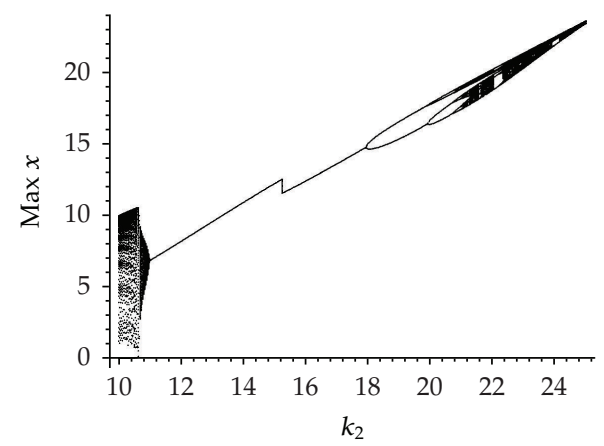

(c)

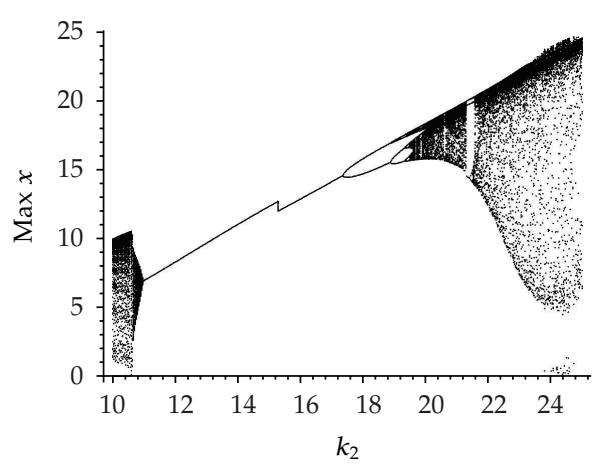

(b)

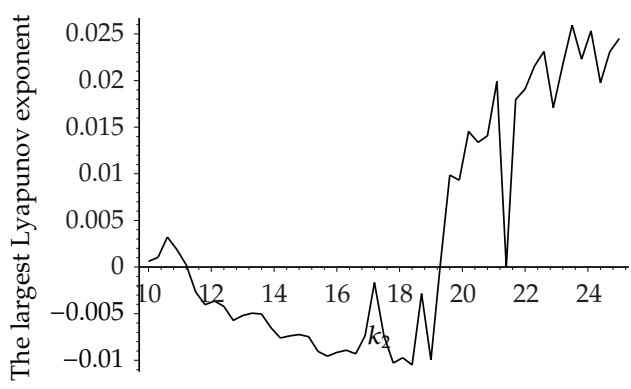

(d)

Figure 1: Bifurcation diagram of system (1.1) with initial conditions $x_{0}=(0.1,0.1,0.1)$. (a) $\varphi=0$; (b) $\varphi=\pi / 2$; (c) $\varphi=\pi$; (d) the largest Lyapunov exponent ( $k_{2}$ from 10 to 25 ) for system (1.1).

and some critical parameters, the diagrams as a function of $k_{2}$ and $\omega$ are obtained for three different cases: $\varphi=0, \pi / 2, \pi$. All other parameters are fixed as follows: $a_{1}=0.8, a_{2}=0.6$, $b_{1}=0.25, b_{2}=2.5, e_{1}=0.45, e_{2}=0.35, c_{1}=0.25, c_{2}=0.55, m_{1}=0.1, m_{2}=0.15, r_{1}=0.8$, $k_{1}=30, \beta=0.35, \varepsilon_{1}=0.5, \varepsilon_{2}=0.1$, and $\varepsilon_{3}=0.5$.

Let us investigate the critical parameter $k_{2}$. Figure 1 shows the bifurcation diagram and the corresponding largest Lyapunov exponents as a function of $k_{2}$ in the range $10 \leq$ $k_{2} \leq 25$ for $\varphi=0, \pi / 2, \pi$ and $\omega=0.3$. It is evident from Figure 1 that the system produces complex dynamics for $10 \leq k_{2} \leq 25$ when $\varphi=0, \pi / 2$ and $\pi$, such as chaotic band, perioddoubling bifurcation, and chaotic band with wide or narrow periodic windows. Nonetheless, it is interesting to notice that the prey $x$ biomass level increases with increase of $k_{2}$, but it can be found that when $\varphi=\pi / 2$, the prey $x$ biomass level is relatively high. Comparison of Figures $1(\mathrm{a}), 1(\mathrm{~b})$, and 1(c) suggests that seasonality may still give a region of periodic solutions for $k_{2}$. The evidence for the cascade of periodic doubling leading to chaos can be seen in Figure 1 for $11 \leq k_{2} \leq 20,11 \leq k_{2} \leq 19.5$, and $11 \leq k_{2} \leq 21.5$. However, Figure 1 (a) shows chaotic bands with a narrow periodic window, and Figures 1(b) and 1(c) show chaotic bands with wide or narrow periodic windows. It is obvious that the seasonal disturbance not only enhances the prey $x$ biomass level, but also changes the complex dynamics when $\varphi=\pi / 2$.

To investigate more carefully the effect of synchronous variation in the intrinsic growth rate and death rate, three strange attractors are plotted for $k_{2}=24$ and three different values 


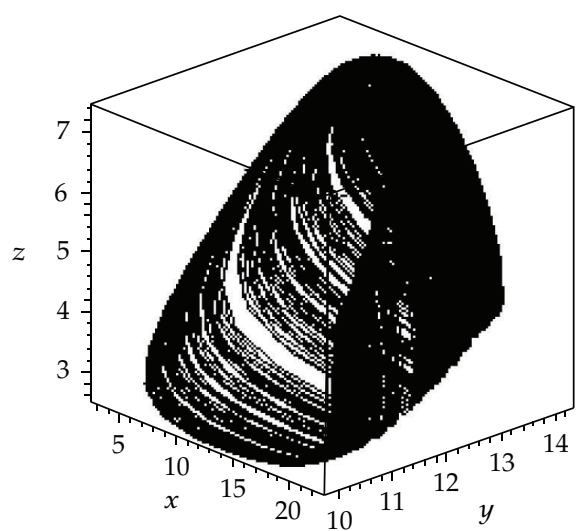

(a)

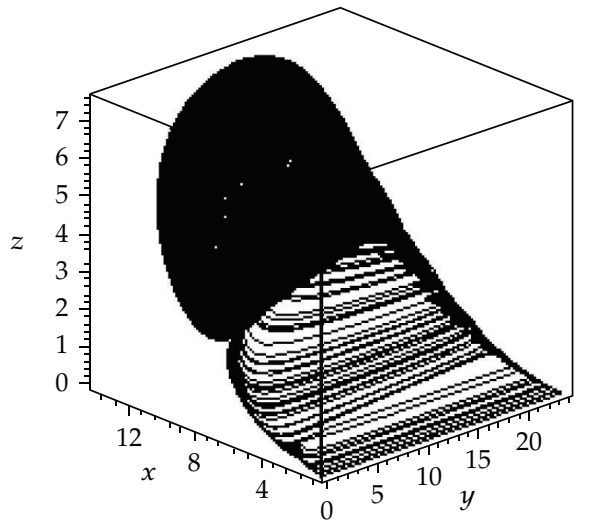

(b)

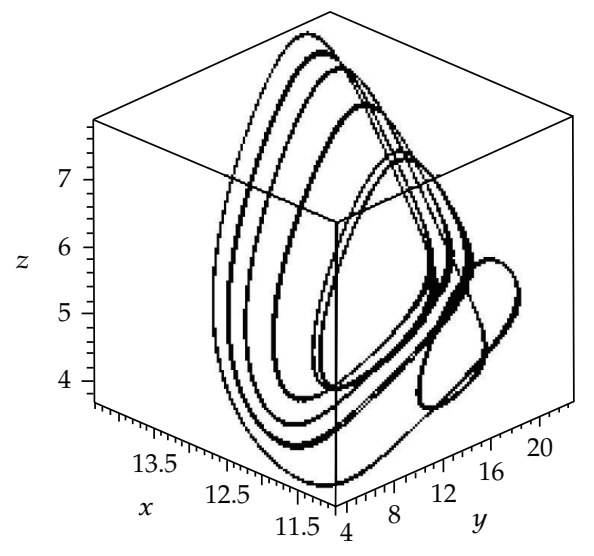

(c)

Figure 2: (a) Chaos when $k_{2}=24, \varphi=0$; (b) chaos when $k_{2}=24, \varphi=(\pi / 2)$; (c) periodic attractor when $k_{2}=24, \varphi=\pi$.

of the synchronous variation parameter: $\varphi=0, \pi / 2, \pi$. Figure 2 shows that when $\varphi$ is 0 or $(\pi / 2)$, system (1.1) has a chaotic attractor at $k_{2}=24$, but when $\varphi=\pi$, system (1.1) has a periodic attractor at $k_{2}=24$. It can be observed from Figure 2 that seasonality in the intrinsic growth rate and death rate can change the chaotic behavior of the system at various values of the synchronous variation $\varphi$.

For further analysis of the effects of seasonality, three chaotic attractors as well as a Poincaré section (the Poincaré section is a classical technique for analyzing dynamic systems $[27,28])$ have been obtained for $k_{2}=23$ and three different values of synchronous variation: $\varphi=0, \pi / 2, \pi$. In this paper, the plane $(z=6)$ was chosen, and values of $y$ were plotted against $x$. The resulting attractors and Poincaré section are shown in Figure 3. Figure 3(a) shows a chaotic attractor at $\varphi=0$, and Figure 3(b) shows a Poincaré section for the chaotic attractor shown in (a). Three wide strips can be seen in Figure 3(b). Figure 3(c) shows a chaotic attractor at $\varphi=\pi / 2$, and Figure 3(d) shows a Poincaré section for the chaotic attractor shown in (c). One wide strip can be seen in Figure 3(d). Figure 3(e) shows a chaotic attractor at $\varphi=\pi$, and Figure 3(f) shows a Poincare section for the chaotic attractor shown in (e). 


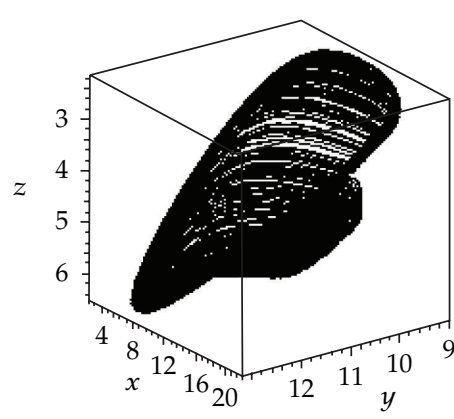

(a)

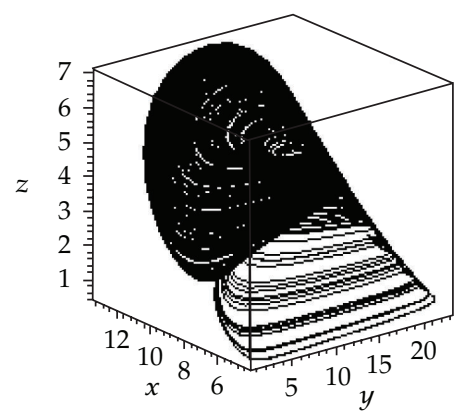

(c)

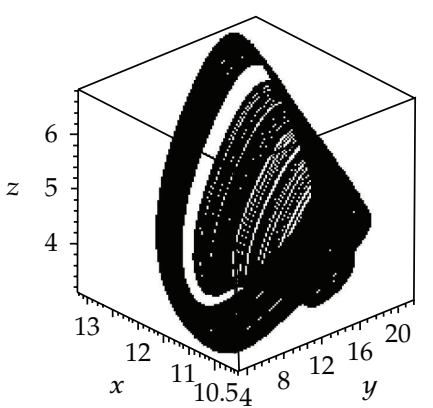

(e)

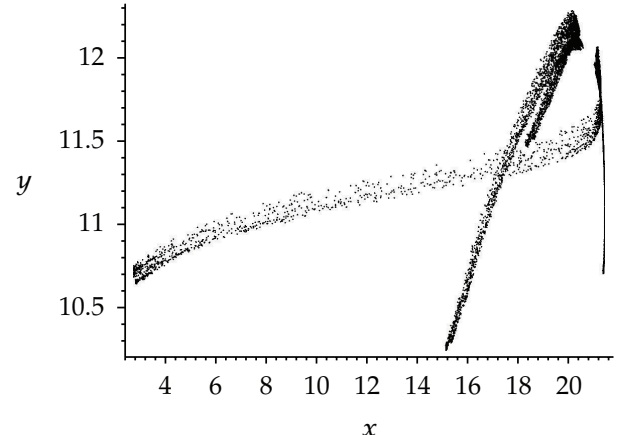

(b)

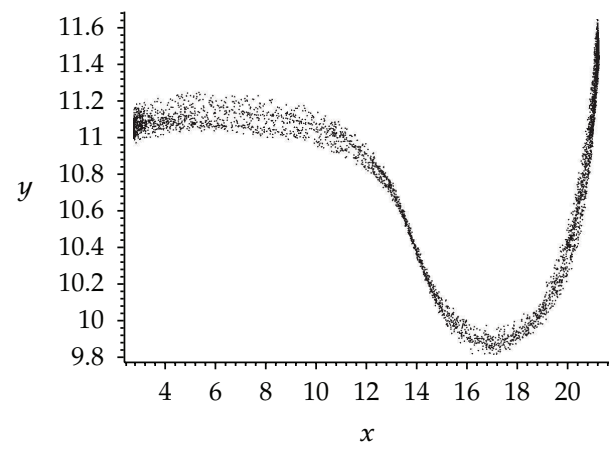

(d)

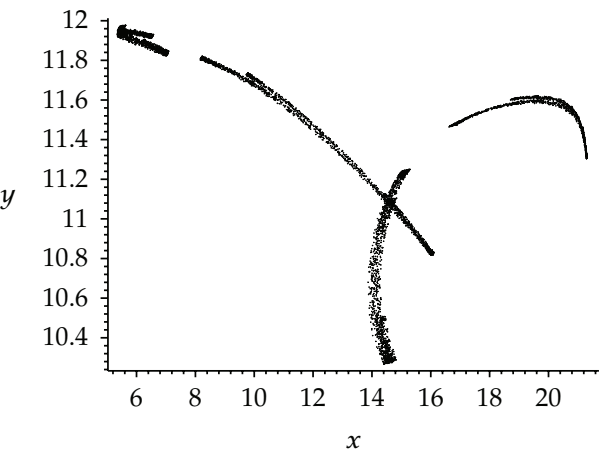

(f)

Figure 3: (a) Strange attractor with $\varphi=0, k_{2}=23$; (b) Poincaré section of attractor (a); (c) strange attractor with $\varphi=(\pi / 2), k_{2}=23$; (d) Poincaré section of attractor $(\mathrm{c})$; (e) strange attractor $\varphi=\pi, k_{2}=23$; (f) Poincare section of attractor (e), showing the values of the states $x$ and $y$ in the plane $z=5$.

Four wide strips can be seen in Figure 3(f). By comparison of the three Poincaré sections, it is apparent that the three chaotic attractors have different structures in the three different cases $\varphi=0, \pi / 2, \pi$. However, they might be better described as stranger chaotic attractors and fractals. These results show that seasonal perturbation can change chaotic attractor structure. In a word, seasonal perturbation is sufficient to change the chaotic attractor trajectory in system (1.1).

Moreover, convincing evidence for deterministic chaos has come from several recent experiments [29-34]. The results of chaos studies have confirmed the importance of detecting 


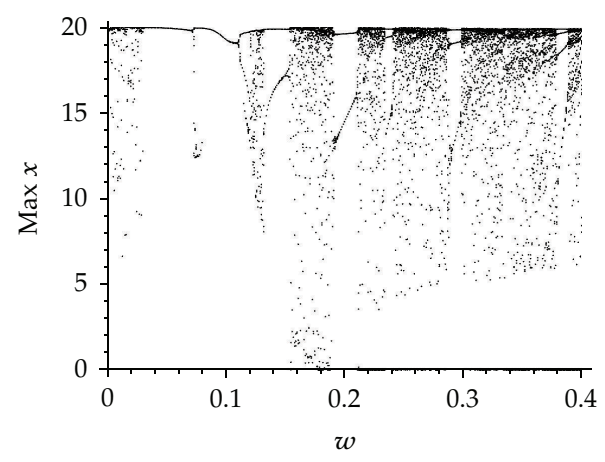

(a)

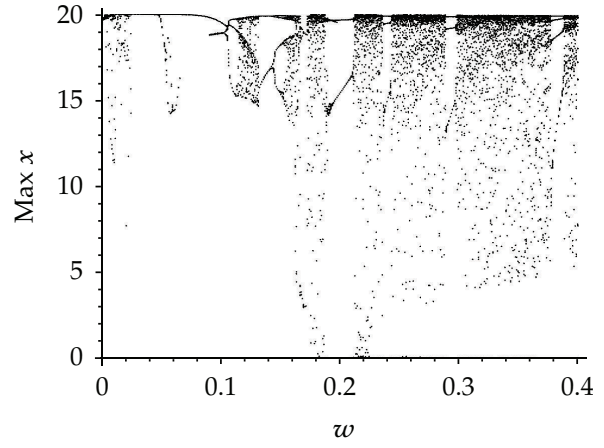

(b)

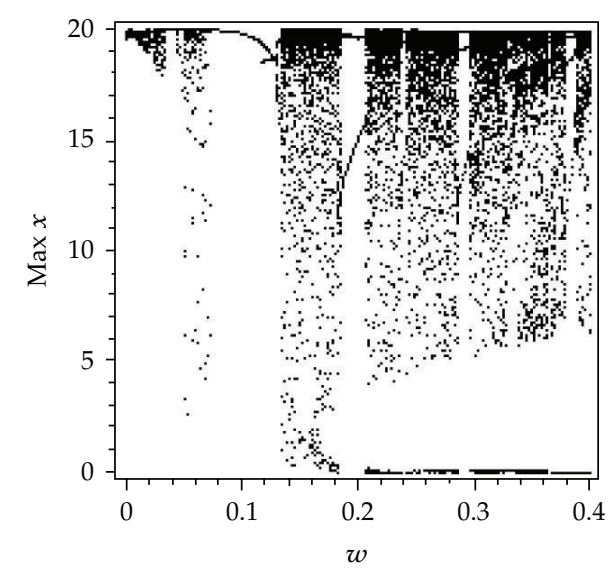

(c)

Figure 4: Bifurcation diagram of system (1.1) with initial conditions $x_{0}=(0.1,0.1,0.1)$. (a) $\varphi=0$; (b) $\varphi=(\pi / 2) ;(\mathrm{c}) \varphi=\pi$.

and exploring chaos. Here, the largest Lyapunov exponents are considered because they have proved to offer the most useful diagnostics for a chaotic system [35-40]. The Lyapunov exponents take into account the average exponential rates of divergence or convergence of nearby orbits in phase space [29]. For a chaotic attractor, the values of the largest Lyapunov exponent must be positive; if they are always negative, chaos cannot be observed, and a stable state or a period attractor will instead appear. In the bifurcation diagram for resource $x$ shown in Figure 1(b), the corresponding largest Lyapunov exponent $10 \leq k_{2} \leq 25$ can be calculated for the system described by (1.1), which is shown in Figure 1(d). It should be stressed that the result is consistent with Figure 1(b), which shows the accuracy and effectiveness of numerical simulation. Moreover, using the simulation of the largest Lyapunov exponents, the existence of chaotic behavior in system (1.1) can be further confirmed.

The other critical parameter to be investigated is $\omega$. Figure 4 depicts the local longterm dynamics maxima for $\varphi=0, \pi / 2, \pi$ and the range $0 \leq \omega \leq 0.4$ and $k_{2}=20$. As $\omega$ increases from 0 to 0.14 , Figure $4(\mathrm{a})$ shows that the system has rich dynamics, including periodic windows, period doubling, chaos, and a chaotic crisis. However, only chaos, chaotic crisis, and periodic windows are observed in Figures $4(\mathrm{~b})$ and 4(c). Figure 4(b) shows that when $\omega$ is slightly increased beyond 0.14 , the size of the chaotic attractor abruptly 
changes, constituting a type of attractor crisis. As $\omega$ continues to increase, a cascade of period-doubling bifurcation occurs, the period- 2 attractor changes to a period- 4 attractor, then a period-8 attractor and period-doubling bifurcation finally leads system (1.1) into chaos. However, only chaos and periodic windows are observed in Figures 4(a) and 4(c). In the range $(0.18,0.4)$, the solution is chaotic with intermittent periodic windows. Similar behavior is observed in Figures 4(a) and 4(c). Hence, it is obvious that the seasonality has a strong effect on the dynamical behaviors of system (1.1) with the synchronous variation $\varphi$ varying.

In order to study the effect of seasonality, three chaotic attractors as well as a Poincare section have been obtained for $\omega=0.26$ and three different values of the synchronous variation parameter: $\varphi=0, \pi / 2, \pi$ and the plane $(z=5)$. The attractors and Poincare sections are shown in Figure 5. Figure 5(a) shows a chaotic attractor at $\varphi=0$, and Figure 5(b) shows a Poincaré section for the chaotic attractor shown in (a). Two wide point regions can be seen in Figure 5(b). Figure 5(c) shows a chaotic attractor at $\varphi=\pi / 2$, and Figure 5(d) shows a Poincaré section for the chaotic attractor shown in (c). Two point regions which are wider than in the Poincaré section shown in (b) can be seen in Figure 5(d). Figure 5(e) shows a chaotic attractor at $\varphi=\pi$, and Figure 5(f) shows a Poincaré section for the chaotic attractor shown in (e). Two point regions which are narrower than in the other two Poincaré sections can be seen in Figure 5(f). By comparison of the three Poincare sections, it is apparent that the three chaotic attractors have different structures in the three different cases $\varphi=0, \pi / 2, \pi$. However, they are all fractals. These results show that the angular frequency of the fluctuations caused by seasonality can change chaotic attractor structure with the synchronous variation $\varphi$ varying.

Based on the above analysis, it can be seen that the seasonal disturbance can enhance the prey $x$ biomass level for $\varphi=\pi / 2$, in which result is agreed with some results in reality. Further, it is also interesting to point out that the seasonality in three different parameters can come into rich and complex dynamical behaviors, but these dynamical behaviors are different. Moreover, the use of mathematical model with seasonality is considered to investigate some biological problems, and the numerical simulation provides an approximation of the real biological system behaviors; hence, these results can promote the study of ecological dynamics.

\section{Conclusions and Remarks}

In this paper, a prey-predator model with Beddington-DeAngelis functional response and seasonal perturbation has been studied analytically and numerically. Mathematical theoretical works have been pursuing the investigation of uniformly persistent, which depicts the threshold expression of some critical parameters and in turn provides a theoretical basis for the numerical simulation. Numerical analysis indicates that the seasonality has a strong effect on the dynamical complexity and species biomass using bifurcation diagrams and Poincare sections. Bifurcation diagrams show that the seasonality in three different parameters can give rise to rich and complex dynamical behaviors, including periodic, period-doubling, period-halving, chaotic crises, and chaos. By comparing the Poincaré sections of various chaotic attractors, it can be determined that these chaotic attractors have different structures with the seasonality in three different parameters. Using numerical simulation of the largest Lyapunov exponents, the existence of chaotic behavior in system (1.1) and the accuracy and effectiveness of numerical simulation can be further confirmed. 


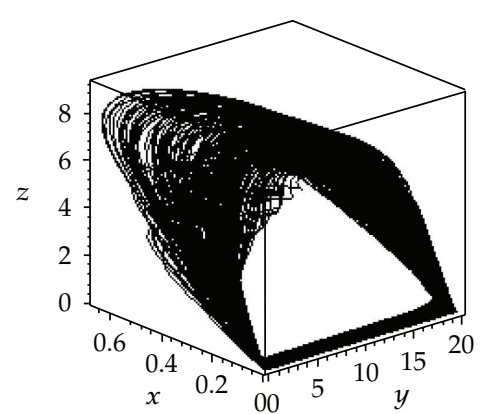

(a)

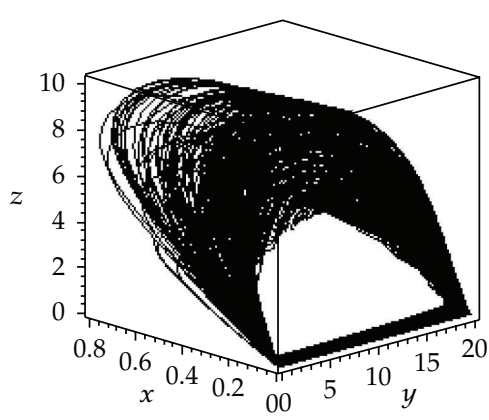

(c)

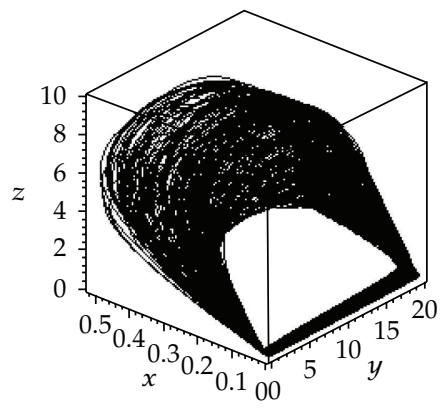

(e)

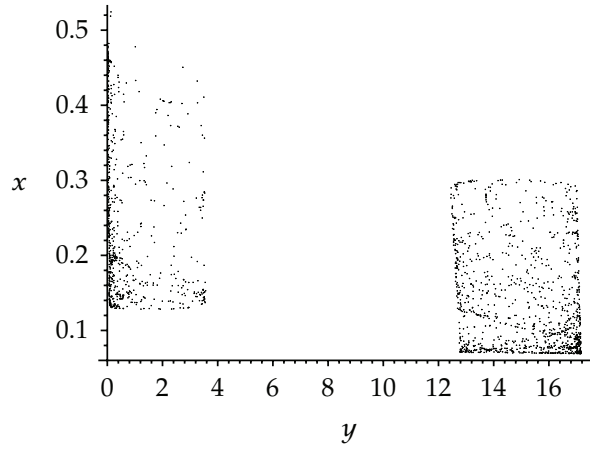

(b)

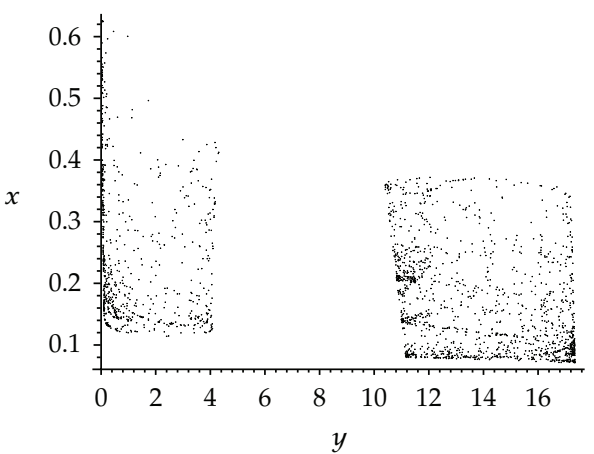

(d)

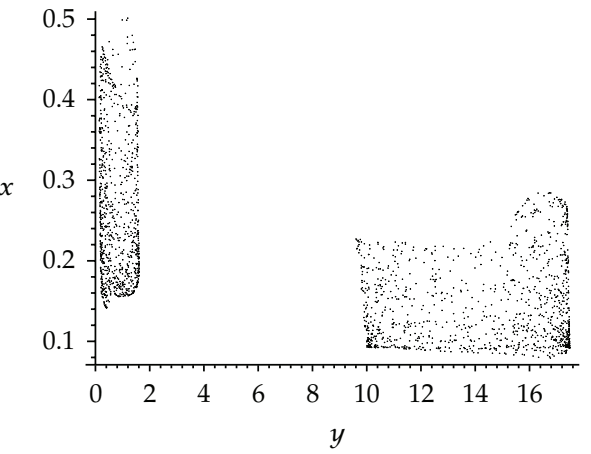

(f)

Figure 5: (a) Strange attractor with $\varphi=0, \omega=0.26$; (b) Poincaré section of attractor (a); (c) strange attractor with $\varphi=(\pi / 2), \omega=0.26$; (d) Poincaré section of attractor (c); (e) strange attractor with $\varphi=\pi, \omega=0.26$; (f) Poincaré section of attractor (e), showing the values of the states $x$ and $y$ in the plane $z=5$.

All these results are expected to be of use in the study of the dynamic complexity of ecosystems.

\section{Acknowledgments}

This work was supported by the National Natural Science Foundation of China (Grant no. 30970305 and Grant no. 31170338) and also by the Zhejiang Provincial Natural Science Foundation of China (Grant no. Y505365). 


\section{References}

[1] S. Gakkhar and R. K. Naji, "Chaos in seasonally perturbed ratio-dependent prey-predator system," Chaos, Solitons and Fractals, vol. 15, no. 1, pp. 107-118, 2003.

[2] R. K. Upadhyay and S. R. K. Iyengar, "Effect of seasonality on the dynamics of 2 and 3 species preypredator systems," Nonlinear Analysis, vol. 6, no. 3, pp. 509-530, 2005.

[3] S. Rinaldi, S. Muratori, and Y. A. Kuznetsov, "Multiple attractors, catastrophes and chaos in seasonally perturbed predator-prey communities," Bulletin of Mathematical Biology, vol. 55, no. 1, pp. 15-35, 1993.

[4] H. Baek, "An impulsive two-prey one-predator system with seasonal effects," Discrete Dynamics in Nature and Society, vol. 2009, Article ID 793732, 19 pages, 2009.

[5] A. Gragnani and S. Rinaldi, "A universal bifurcation diagram for seasonally perturbed predator-prey models," Bulletin of Mathematical Biology, vol. 57, no. 5, pp. 701-712, 1995.

[6] H. G. Yu, S. M. Zhong, R. P. Agarwal, and S. K. Sen, "Effect of seasonality on the dynamical behavior of an ecological system with impulsive control strategy," Journal of the Franklin Institute, vol. 348, no. 4, pp. 652-670, 2011.

[7] T. S. Parker and L. O. Chua, Practical Numerical Algorithms for Chaotic System, Springer, New York, NY, USA, 1989.

[8] W. M. Schaffer, "Order and chaos in ecological systems,” Ecology, vol. 66, no. 1, pp. 93-106, 1985.

[9] R. K. Upadhyay and V. Rai, "Crisis-limited chaotic dynamics in ecological systems," Chaos, Solitons and Fractals, vol. 12, no. 2, pp. 205-218, 2001.

[10] S. J. Lv and M. Zhao, "The dynamic complexity of a three species food chain model," Chaos, Solitons and Fractals, vol. 37, no. 5, pp. 1469-1480, 2008.

[11] S. Gakkhar and R. K. Naji, "Order and chaos in a food web consisting of a predator and two independent preys," Communications in Nonlinear Science and Numerical Simulation, vol. 10, no. 2, pp. 105-120, 2005.

[12] F. Y. Wang, C. P. Hao, and L. S. Chen, "Bifurcation and chaos in a Monod-Haldene type food chain chemostat with pulsed input and washout," Chaos, Solitons and Fractals, vol. 32, no. 1, pp. 181-194, 2007.

[13] Y. K. Xue and X. F. Duan, "The dynamic complexity of a Holling type-IV predator-prey system with stage structure and double delays," Discrete Dynamics in Nature and Society, vol. 2011, Article ID 509871, 19 pages, 2011.

[14] M. Zhao and S. J. Lv, "Chaos in a three-species food chain model with a Beddington-DeAngelis functional response," Chaos, Solitons and Fractals, vol. 40, no. 5, pp. 2305-2316, 2009.

[15] S. Gakkhar and R. K. Naji, "Seasonally perturbed prey-predator system with predator-dependent functional response," Chaos, Solitons and Fractals, vol. 18, no. 5, pp. 1075-1083, 2003.

[16] T. W. Hwang, "Uniqueness of limit cycles of the predator-prey system with Beddington-DeAngelis functional response," Journal of Mathematical Analysis and Applications, vol. 290, no. 1, pp. 113-122, 2004.

[17] N. Zhang, F. D. Chen, Q. Q. Su, and T. Wu, "Dynamic behaviors of a harvesting Leslie-Gower predator-prey model," Discrete Dynamics in Nature and Society, vol. 2011, Article ID 473949, 14 pages, 2011.

[18] V. Křivan and J. Eisner, "The effect of the Holling type II functional response on apparent competition," Theoretical Population Biology, vol. 70, no. 4, pp. 421-430, 2006.

[19] X. Li and W. Yang, "Permanence of a discrete predator-prey systems with Beddington-deAngelis functional response and feedback controls," Discrete Dynamics in Nature and Society, vol. 2008, Article ID 149267, 8 pages, 2008.

[20] R. X. Wu and L. Li, "Permanence and global attractivity of discrete predator-prey system with hassellvarley type functional response," Discrete Dynamics in Nature and Society, vol. 2009, Article ID 323065 , 17 pages, 2009.

[21] L. J. Chen, J. Y. Xu, and Z. Li, "Permanence and global attractivity of a delayed discrete predator-prey system with general holling-type functional response and feedback controls," Discrete Dynamics in Nature and Society, vol. 2008, Article ID 629620, 17 pages, 2008.

[22] J. R. Beddington, "Mutual interference between parasites or predator and its effect on searching efficiency," Journal of Animal Ecology, vol. 44, no. 1, pp. 331-340, 1975.

[23] D. L. DeAngelis, R. A. Goldstein, and R. V. Neill, "A model for trophic interaction," Ecology, vol. 56, no. 4 , pp. 881-892, 1975.

[24] H. Baek, "Species extinction and permanence of an impulsively controlled two-prey one-predator system with seasonal effects," BioSystems, vol. 98, no. 1, pp. 7-18, 2009. 
[25] S. Gakkhar and R. K. Naji, "Seasonally perturbed prey-predator system with predator-dependent functional response," Chaos, Solitons and Fractals, vol. 18, no. 5, pp. 1075-1083, 2003.

[26] K. Wang, "Permanence and global asymptotical stability of a predatorprey model with mutual interference," Nonlinear Analysis: Real World Applications, vol. 12, no. 2, pp. 1062-1071, 2011.

[27] F. D. Chen, "On a nonlinear nonautonomous predator-prey model with diffusion and distributed delay," Journal of Computational and Applied Mathematics, vol. 180, no. 1, pp. 33-49, 2005.

[28] R. Barrio, "Sensitivity tools vs. Poincaré sections," Chaos, Solitons and Fractals, vol. 25, no. 3, pp. 711726, 2005.

[29] C. Masoller, A. C. S. Schifino, and L. Romanelli, "Characterization of strange attractors of lorenz model of general circulation of the atmosphere," Chaos, Solitons and Fractals, vol. 6, pp. 357-366, 1995.

[30] M. Zhao and L. M. Zhang, "Permanence and chaos in a host-parasitoid model with prolonged diapause for the host," Communications in Nonlinear Science and Numerical Simulation, vol. 14, no. 12, pp. 4197-4203, 2009.

[31] M. Hagmüller and G. Kubin, "Poincaré pitch marks," Speech Communication, vol. 48, no. 12, pp. 1650$1665,2006$.

[32] H. G. Yu, M. Zhao, S. J. Lv, and L. L. Zhu, "Dynamic complexities in a parasitoid-host-parasitoid ecological model," Chaos, Solitons and Fractals, vol. 39, no. 1, pp. 39-48, 2009.

[33] J. G. Sportt, Chaos and Time-Series Analysis, Oxford University Press, New York, NY, USA, 2003.

[34] M. T. Rosenstein, J. J. Collins, and C. J. de Luca, "A practical method for calculating largest Lyapunov exponents from small data sets," Physica D, vol. 65, no. 1-2, pp. 117-134, 1993.

[35] S. J. Lv and M. Zhao, "The dynamic complexity of a host-parasitoid model with a lower bound for the host," Chaos, Solitons and Fractals, vol. 36, no. 4, pp. 911-919, 2008.

[36] Q. Jia, "Hyperchaos generated from the Lorenz chaotic system and its control," Physics Letters, Section A, vol. 366, no. 3, pp. 217-222, 2007.

[37] M. Zhao, L. M. Zhang, and J. Zhu, "Dynamics of a host-parasitoid model with prolonged diapause for parasitoid," Communications in Nonlinear Science and Numerical Simulation, vol. 16, no. 1, pp. 455-462, 2011.

[38] M. Zhao, H. G. Yu, and J. Zhu, "Effects of a population floor on the persistence of chaos in a mutual interference host-parasitoid model," Chaos, Solitons and Fractals, vol. 42, no. 2, pp. 1245-1250, 2009.

[39] F. Grond, H. H. Diebner, S. Sahle, A. Mathias, S. Fischer, and O. E. Rossler, "A robust, locally interpretable algorithm for Lyapunov exponents," Chaos, Solitons and Fractals, vol. 16, no. 5, pp. 841852, 2003.

[40] L. L. Zhu and M. Zhao, "Dynamic complexity of a host-parasitoid ecological model with the Hassell growth function for the host," Chaos, Solitons and Fractals, vol. 39, no. 1, pp. 1259-1269, 2009. 


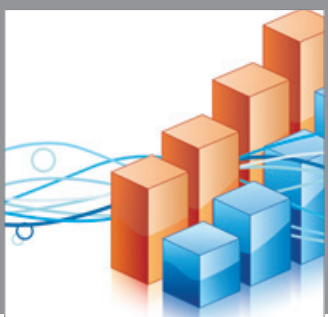

Advances in

Operations Research

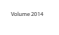

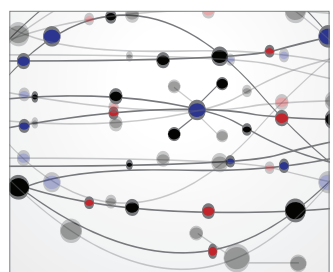

\section{The Scientific} World Journal
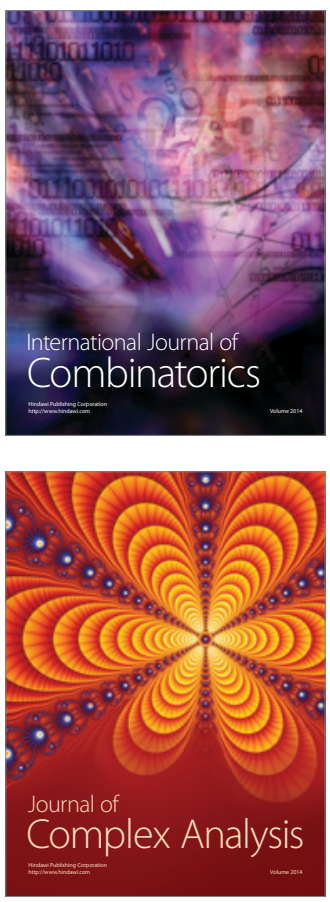

International Journal of

Mathematics and

Mathematical

Sciences
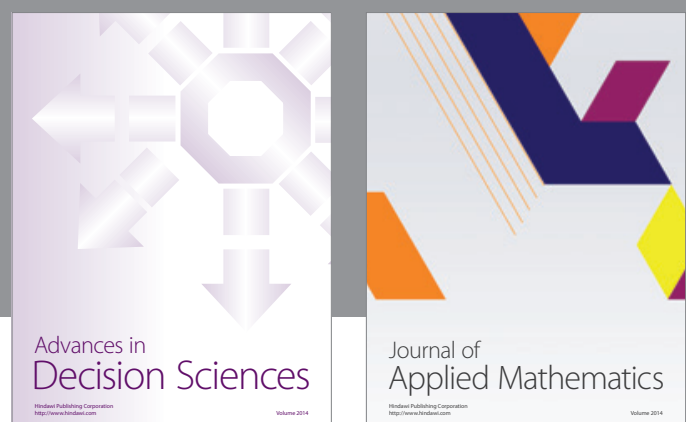

Journal of

Applied Mathematics
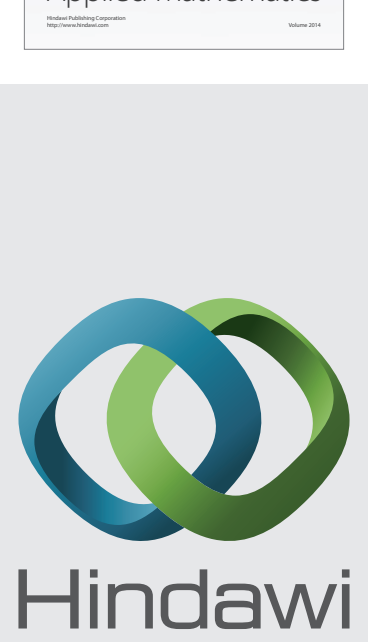

Submit your manuscripts at http://www.hindawi.com
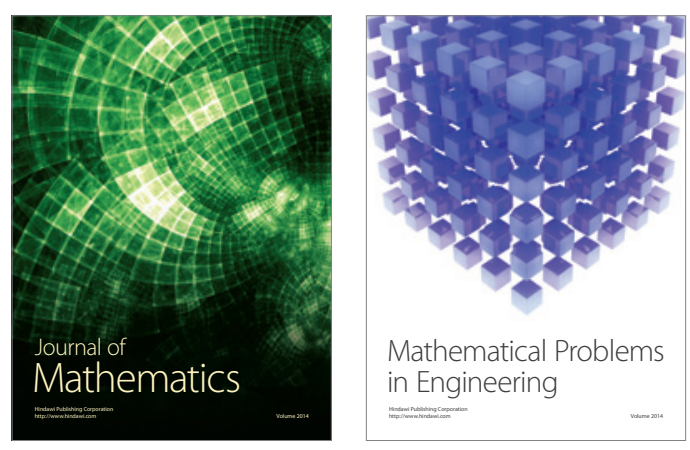

Mathematical Problems in Engineering
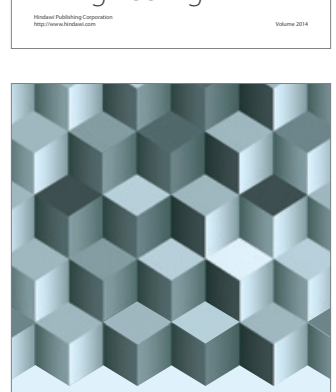

Journal of

Function Spaces
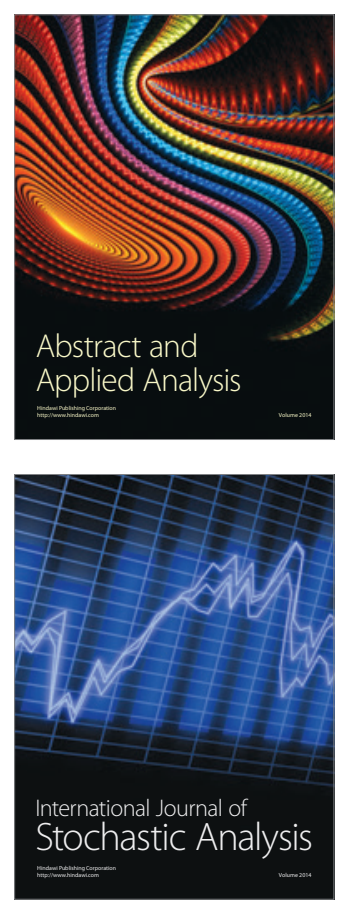

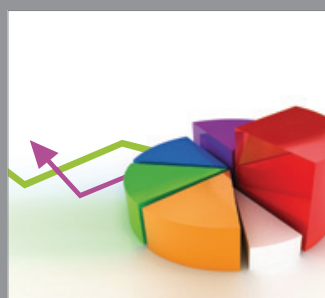

ournal of

Probability and Statistics

Promensencen
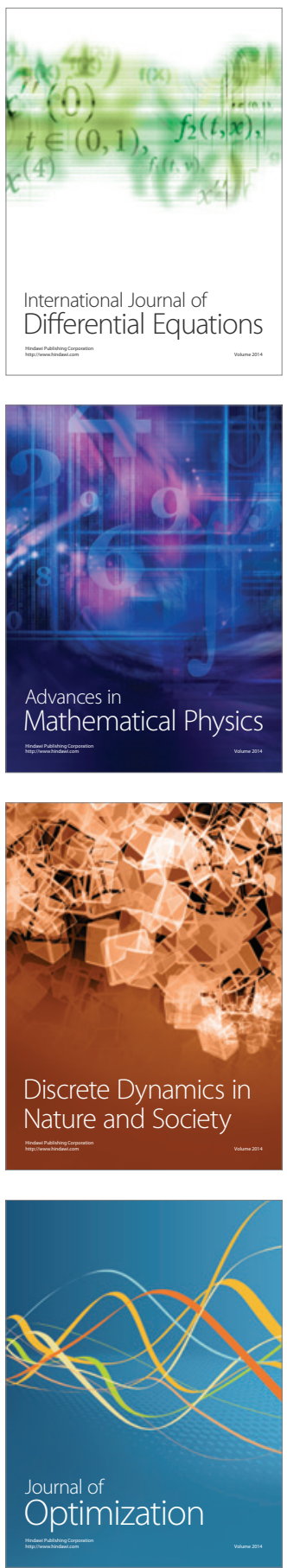